\title{
Early-onset obesity and paternal 2 pter deletion encompassing the ACP1, TMEM18, and MYT1L genes
}

\author{
Martine Doco-Fenzy ${ }^{\star}, 1$ Camille Leroy ${ }^{1}$, Anouck Schneider ${ }^{2}$, Florence Petit ${ }^{3}$, Marie-Ange Delrue ${ }^{4}$, \\ Joris Andrieux ${ }^{3}$, Laurence Perrin-Sabourin ${ }^{5}$, Emilie Landais ${ }^{1}$, Azzedine Aboura ${ }^{5}$, Jacques Puechberty ${ }^{2,6}$, \\ Manon Girard ${ }^{6}$, Magali Tournaire ${ }^{6}$, Elodie Sanchez ${ }^{2}$, Caroline Rooryck ${ }^{4}$, Agnès Ameil ${ }^{7}$, \\ Michel Goossens ${ }^{8}$, Philippe Jonveaux ${ }^{9}$, Geneviève Lefort ${ }^{2,6}$, Laurence Taine ${ }^{4}$, Dorothée Cailley ${ }^{4}$, \\ Dominique Gaillard ${ }^{1}$, Bruno Leheup ${ }^{10}$, Pierre Sarda ${ }^{2}$ and David Geneviève ${ }^{2}$
}

Obesity is a common but highly, clinically, and genetically heterogeneous disease. Deletion of the terminal region of the short arm of chromosome 2 is rare and has been reported in about 13 patients in the literature often associated with a Prader-Willi-like phenotype. We report on five unrelated patients with 2 p25 deletion of paternal origin presenting with earlyonset obesity, hyperphagia, intellectual deficiency, and behavioural difficulties. Among these patients, three had de novo pure 2 pter deletions, one presented with a paternal derivative $\operatorname{der}(2) \mathrm{t}(2 ; 15)(\mathrm{p} 25.3 ; \mathrm{q} 26)$ with deletion in the 2 pter region and the last patient presented with an interstitial 2p25 deletion. The size of the deletions was characterized by SNP array or array-CGH and was confirmed by fluorescence in situ hybridization (FISH) studies. Four patients shared a 2 p25.3 deletion with a minimal critical region estimated at $1.97 \mathrm{Mb}$ and encompassing seven genes, namely SH3HYL1, ACP1, TMEMI8, SNTG2, TPO, PXDN, and MYT1L genes. The fifth patient had a smaller interstitial deletion encompassing the TPO, PXDN, and MYT1L genes. Paternal origin of the deletion was determined by genotyping using microsatellite markers. Analysis of the genes encompassed in the deleted region led us to speculate that the ACP1, TMEM18, and/or MYT1L genes might be involved in early-onset obesity. In addition, intellectual deficiency and behavioural troubles can be explained by the heterozygous loss of the SNTG2 and MYT1L genes. Finally, we discuss the parent-of-origin of the deletion.

European Journal of Human Genetics (2014) 22, 471-479; doi:10.1038/ejhg.2013.189; published online 16 October 2013

Keywords: deletion 2p25.3; obesity; abnormal behaviour; TMEM18; MYT1L

\section{INTRODUCTION}

Pure distal deletions of the short arm of chromosome 2 are rare and to the best of our knowledge $<20$ patients have been reported in the literature. ${ }^{1-8}$ Becker et al $l^{6}$ reported a patient with a de novo pure $2 \mathrm{p} 25.2$ deletion, bilateral severe talipes equinovarus, pulmonary valve stenosis, nasal polyps, mild psychomotor retardation, and overweightness with food seeking behaviour. Several patients were then reported presenting with obesity, intellectual disability (ID), and 2p25 deletion. A PraderWilli-like phenotype was thus defined for 2p25 deletion (early-onset obesity, pervasive developmental disorder, and behavioural problems). Stevens et $a l^{7}$ reported three adult siblings and three unrelated patients with ID, obesity or overweight, and/or square-shaped stature and pure terminal or interstitial 2p25.3 deletion and defined the length of the lost region by SNP array or oligoarray-CGH. Recently, Rio et al ${ }^{8}$ reported a sporadic case (Twin 1) with 2p25.3 interstitial deletion and ID, obesity, and behavioural troubles. Other patients reported in the literature, with larger deletions of the short arm of chromosome 2, interstitial deletions or large deletions combined with other large rearrangements have heterogeneous phenotypes.
We report on five new unrelated patients with early-onset obesity, behavioural difficulties, and paternal 2p25.3 deletion, in which the deletions were characterized by SNP array or array-CGH, and discuss the potential effects of the deleted genes.

\section{PATIENTS AND METHODS}

Clinical reports

All five patients were examined by at least one of the authors and gave their informed consent for the analysis and for the publication of the pictures. Front and lateral views of the patients as well as growth curves are reported in Figure 1.

Patient 1. The patient was the first child born to unrelated healthy parents at 36 WG. Birth parameters were normal (length: $48 \mathrm{~cm}$, weight: $3170 \mathrm{~g}$, and OFC: $34 \mathrm{~cm}$ ). Her father and mother were of normal height (both measured $166 \mathrm{~cm}$ ) and normal weight. The newborn girl presented with severe hypotonia and failure to thrive. She had small hands and feet and bilateral genu valgum. She developed mild developmental delay, sat unaided at 11 months, and walked at 24 months. She was overweight from 19 months of age on. At 33 months, she weighed $21 \mathrm{~kg}(+6 \mathrm{SD})$, her height was $95 \mathrm{~cm}(+1.5 \mathrm{SD})$, and OFC was $49.5 \mathrm{~cm}$ ( $+1 \mathrm{SD}$ ). At 4 years, she had severe obesity (weight: $32 \mathrm{~kg}$,

\footnotetext{
${ }^{1}$ Service de Génétique, Hôpital Maison-Blanche, CHRU, UFR de Médecine, Reims, France; ²Département de Génétique Médicale, CHRU Montpellier, Faculté de Médecine de Montpellier-Nimes, Université Montpellier 1, Montpellier, France; ${ }^{3}$ Service de Génétique, CHU de Lille, Lille, France; ${ }^{4}$ Service de Génétique Médicale, Laboratoire MRGM, (EA 4576), CHRU de Bordeaux, Bordeaux, France; ${ }^{5}$ Fédération de Génétique, Hopital Robert Debré, APHP Paris, Paris, France; 6 Plateforme puce à ADN, CHRU Montpellier, Université Montpellier 1, Montpellier, France; ${ }^{7}$ Service de Pédiatrie, American Memorial Hospital, CHRU, Reims, France; ${ }^{8}$ Laboratoire de Génétique, AP-HP, et INSERM U-841, CHU Henri Mondor, Créteil, France; '9Service de Génétique, CHRU de Nancy-Brabois, Inserm U954, Université de Lorraine, Nancy, France; ${ }^{10} \mathrm{CHU}$ de Nancy, Pole Enfant, Service de génétique clinique, Vandoeuvre les nancy, F-54511, France

${ }^{*}$ Correspondence: Professor M Doco-Fenzy, Service de Génétique, Hôpital Maison-Blanche, 45 rue Cognacq Jay, CHU-Reims, EA3801, Reims Cedex 51092, France. Tel: +33 326788582; Fax: +33 326784145; E-mail: mdocofenzy@chu-reims.fr
}

Received 22 February 2013; revised 24 June 2013; accepted 24 July 2013; published online 16 October 2013 
a

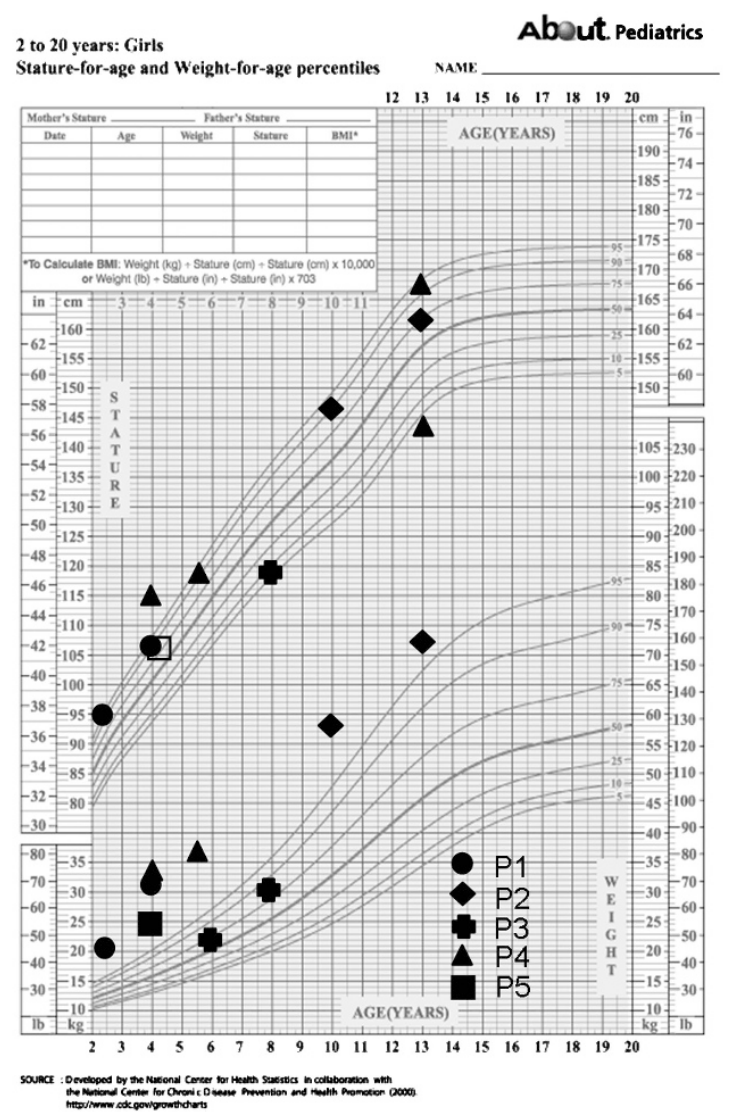

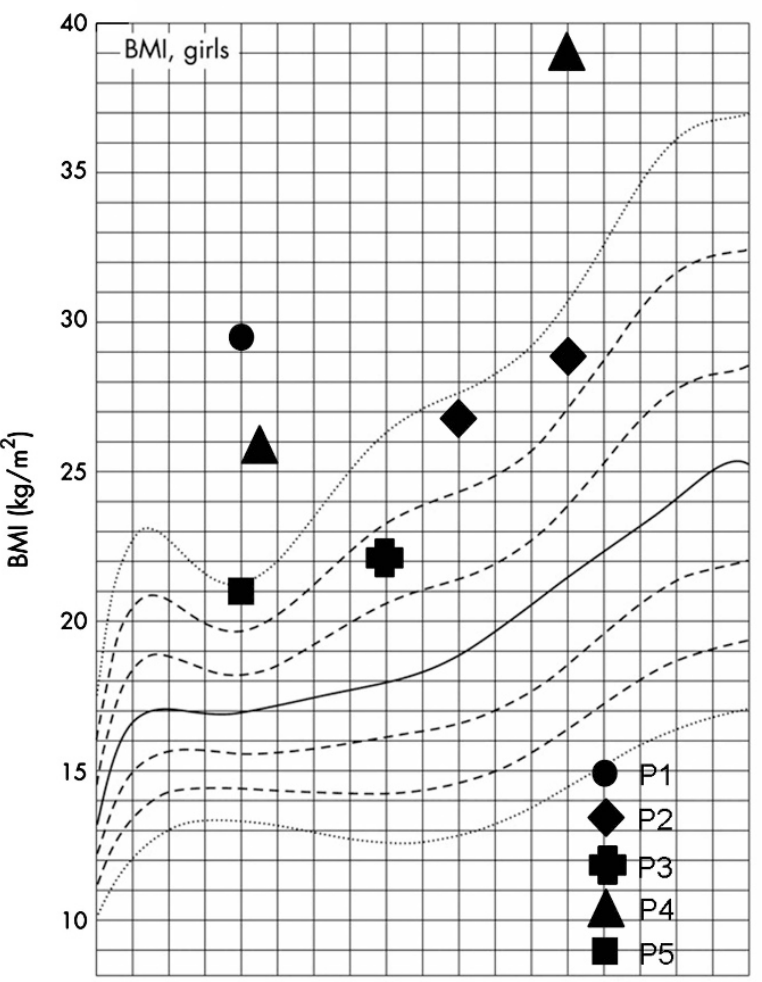

$\begin{array}{lllllllllllllllll}0 & 1 & 2 & 3 & 4 & 5 & 6 & 7 & 8 & 9 & 1011 & 121314 & 15161718\end{array}$ Age (years)

\section{b}
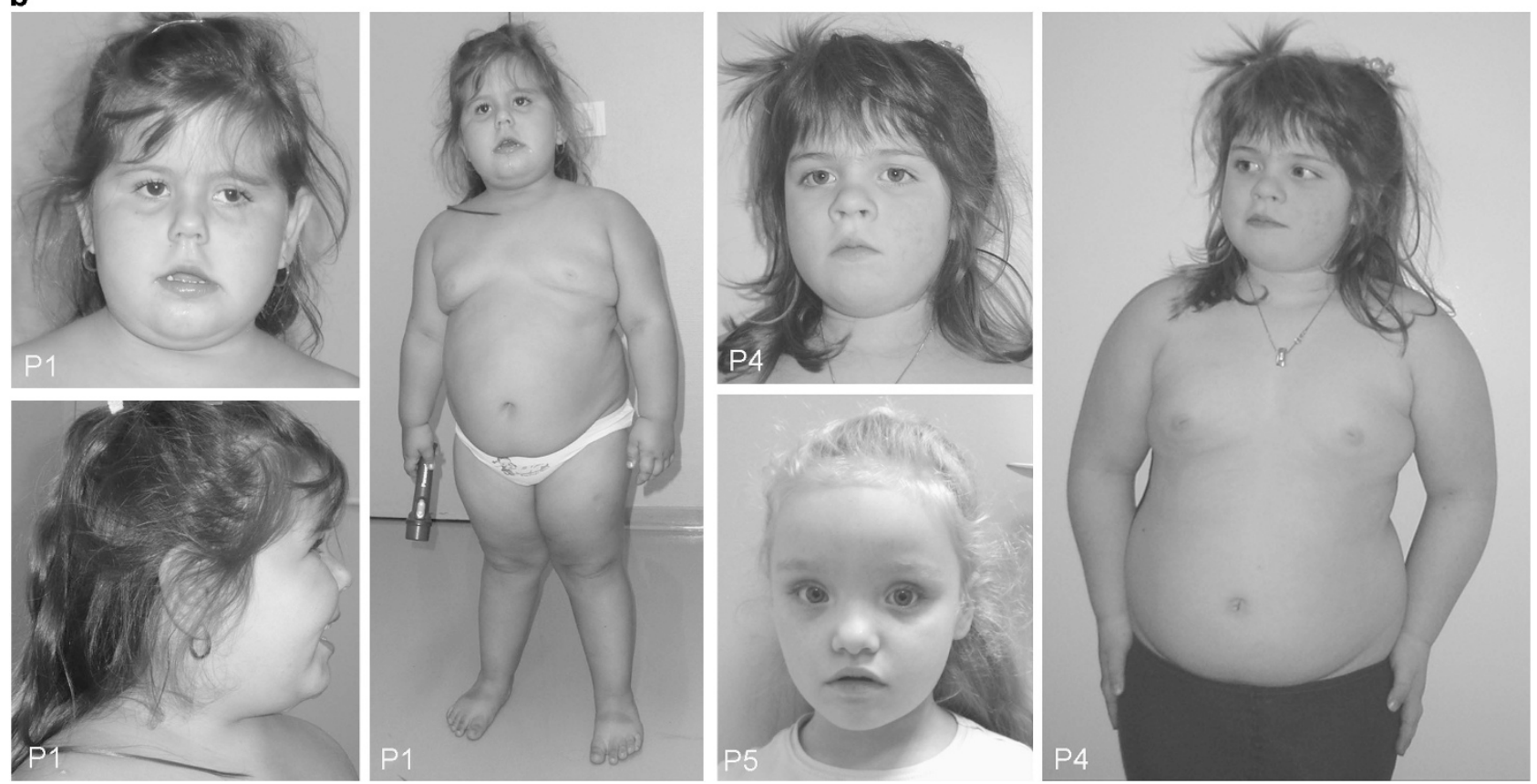

Figure 1 (a) On the left growth curves for patients 1-5 showing the early-onset obesity and on the right BMI graphs for patients 1-5. (b) Pictures of patients 1 and 4-5 showing the facial features. Patients 1 and 4 have thin eyebrows, downslanting palpebral fissures, small nose with upturned nostrils, small mouth, thin lips, and obesity. Patient 2 has horizontal palpebral fissures, frontal bossing, short philtrum, and everted upper lip.

+9 SD; height: $106 \mathrm{~cm},+2$ SD; OFC: $51 \mathrm{~cm},+1$ SD and BMI: $28.5,+5.5 \mathrm{SD})$. She presented with language delay with only 30 words and abnormal behaviour, including heteroagressivity towards other children, outbursts, and hyperphagia. She also manifested severe sleep disturbances possibly due to sleep apnoea. She had distinct facial features namely a round face, full cheeks, thin eyebrows, downslanting palpebral fissures, small nose 
with upturned nostrils and short columella, small mouth with downslanting commissures, large ears, and a low hairline. At 6 years of age, she attended a special school.

Skeletal X-rays and brain MRI were normal. Thyroid screening showed a moderate elevation of TSH: $4.75 \mu \mathrm{IU} / \mathrm{ml} \quad(N$ : 0.27-4.2) but normal T4: $15.99 \mathrm{pmol} / \mathrm{l}(\mathrm{N}: 12-22)$ and T3: $4.67 \mathrm{pg} / \mathrm{ml}$ (N: 2-34.4). Plasmatic leptin level was high $(32.84 \mathrm{ng} / \mathrm{ml} ; \mathrm{N}: 1.5-6)$. Urinary MPS/OS, plasmatic CAA, insulin T0: $5.6 \mu \mathrm{IU} / \mathrm{ml}(N<15)$, FSH, LH, IGF1, and IGFBP3 were normal.

Patient 2. Patient 2 was born at $38 \mathrm{WG}$ to unrelated healthy parents after an uneventful pregnancy. Birth parameters were normal (weight: $3420 \mathrm{~g}$ and length: $51 \mathrm{~cm}$ ). She had psychomotor delay (walked unaided at 24 months and had delayed language with two words at 4 years of age).

She had seizures until 4 years of age. She presented with anxiety, hyperphagia, and behavioural problems, namely aggressive behaviour towards other children, stereotypies, hyperactivity, and cries. She needed special schooling at the age of 4 years. She was first seen in the Genetics Department at the age of 10 years. Her weight was $57 \mathrm{~kg}(>+4 \mathrm{SD})$, height $146 \mathrm{~cm}$ $(+1.5 \mathrm{SD})$, OFC $54.5 \mathrm{~cm}(+2 \mathrm{SD})$, and BMI 26.74 (around $+4.5 \mathrm{SD}$ ). She had distinct facial features, namely slight hypertelorism, bilateral epicanthal folds with almond-shaped palpebral fissures, small ears with large lobules, strabismus, thick lips, and a high-arched palate. At the age of 13 years, her weight was $73 \mathrm{~kg}(>+4 \mathrm{SD})$, height $161 \mathrm{~cm}(+1.2 \mathrm{SD})$, and BMI 28.16 (around $+3.7 \mathrm{SD}$ )

Brain MRI, skeletal X-rays, kidney ultrasounds, metabolic and genetic investigations were normal. Thyroid function was also normal (TSH: $2.83 \mu \mathrm{IU} / \mathrm{ml}(N: 0.50-6.00), \mathrm{T} 4: 17.0 \mathrm{pmol} / \mathrm{l}(N: 11.00-21.00)$, and T3: $6.8 \mathrm{pg} / \mathrm{ml}(\mathrm{N}: 4.0-8.0))$. Fundus examination revealed pigmentary changes.

Patient 3. This patient was born at $40 \mathrm{WG}$ to unrelated healthy parents. Birth parameters were normal (weight: $2890 \mathrm{~g}$, length: $47.5 \mathrm{~cm}$, and OFC: $34 \mathrm{~cm}$ ). The baby boy walked alone at 20 months. He had psychomotor delay, verbal dyspraxia, and cognitive delay at 3 years of age. At the age of 8 years, he could not read or write and needed special schooling. He had normal behaviour except that he ate large quantities of food. At the age of 6 years, his weight was $21.3 \mathrm{~kg}(+1 \mathrm{SD})$. He was still overweight at 8 years with weight: $30.8 \mathrm{~kg}$ ( $+1.5 \mathrm{SD})$, height: $118 \mathrm{~cm}(-1.5 \mathrm{SD})$, OFC: $52 \mathrm{~cm}(-0.5 \mathrm{SD})$, and BMI: 22.3 $(+3 \mathrm{SD})$. He had bilateral third and fourth toe clinodactyly. He had no distinct facial features except strabismus. Hands and feet were also normal.

Cerebral EEG showed left rolandic spikes. Brain MRI, metabolic, and genetic investigations were normal. Thyroid function was also normal

Patient 4. The patient was the only child of healthy unrelated Caucasian parents with a history of two first-trimester miscarriages. The father and mother were of normal height (166 and $160 \mathrm{~cm}$, respectively) and weight. Pregnancy was uneventful. The baby girl was born at term with normal parameters (length: $49.5 \mathrm{~cm}$, weight: $3110 \mathrm{~g}$, and OFC: $33.5 \mathrm{~cm}$ ). She had earlyonset obesity: weight was $15 \mathrm{~kg}(+2 \mathrm{SD})$ at 2 years of age. At the age of 3 years, she showed speech delay (first words at 15 months and association of two words at 26 months). She also presented with hyperphagia and behavioural problems, namely aggressive behaviour towards other children, stereotypies, hyperactivity, unexplained cries and laughs, sleep disturbances, self-injury, and head shaking. At 4 years 10 months of age, she measured $115.1 \mathrm{~cm}(+2.5 \mathrm{SD})$, weighed $33.8 \mathrm{~kg}$, OFC was $52.5 \mathrm{~cm}(+2 \mathrm{SD})$ and BMI was $25.5(+5 \mathrm{SD})$. She had distinct facial features including an asymmetrical round face, full cheeks, thin eyebrows, downslanting palpebral fissures, small nose with upturned nostrils and short columella, small mouth, thin lips, large and thick ears, and a low hairline. Hands and feet were normal. At 5.5 years of age, height was $119 \mathrm{~cm}(+1 \mathrm{SD})$ and weight was $37 \mathrm{~kg}(>+4 \mathrm{SD})$. She attended school for children with special needs. At the age of 6 years, she developed hypothyroidism and was treated with levothyrox. She had menses at 10 years. At 13 years, she measured $167 \mathrm{~cm}(+2 \mathrm{SD})$ and weighed $109 \mathrm{~kg}$ $(>+4 \mathrm{SD})$, BMI was $39(>+4 \mathrm{SD})$ with troncular obesity and hirsutism. She has a happy disposition and is extraverted and disinhibited.

Cholesterol and triglycerides, electroencephalogram, and brain MRI were all normal. Standard blood chromosomes of the affected child and her parents were normal as well as screening for Prader-Willi and fragile-X syndrome mutation.

Patient 5. The patient was a girl, the product of a trichorionic triamniotic pregnancy obtained by ICSI after 12 years of infertility. This was the first pregnancy of healthy unrelated Caucasian parents. She was born at 31 weeks of gestation by caesarean section. At birth, weight was $2090 \mathrm{~g}$ ( + 3 SD), length $44 \mathrm{~cm}(+1 \mathrm{SD})$, and head circumference $31 \mathrm{~cm}(+1 \mathrm{SD})$. There were no feeding difficulties during the neonatal period. She was referred to the geneticist at the age of 4 years because of mild developmental delay (sat at 13 months and walked at 21 months). Obesity developed between 2.5 and 3 years of age. She had speech delay, associated two words at 3 years, and made short sentences at 4 years. IQ was 70 . She had behavioural troubles with motor stereotypies, palmar and plantar hypersensitivity, and hyperphagia with a seemingly insatiable appetite. At the age of 4 years, weight was $24 \mathrm{~kg}(+4 \mathrm{SD})$, height $107 \mathrm{~cm}(+1 \mathrm{SD})$, OFC $52 \mathrm{~cm}(+1.5 \mathrm{SD})$, and BMI 21 (+3 SD). She attended nursery school with an auxiliary. She had distinct facial features with brachycephaly, high forehead, hypertelorism, epicanthus, intermittent divergent strabismus, and a short philtrum. Hands and feet were short, with bilateral fifth digit clinodactyly and dystrophic toenails.

Brain MRI, standard blood chromosomes, and thyroid function were normal (TSH: $4.45 \mu \mathrm{IUml}(N$ : 0.35-4.94), T4: $17.38 \mathrm{pmol} / \mathrm{l}$ ( $N$ : 9.00-19.00), and T3: $4.18 \mathrm{pg} / \mathrm{ml}(\mathrm{N}: 2.47-4.10))$

\section{Methods}

The required consent was obtained from patients or their parents for all samples analysed.

DNA extraction. DNA was extracted from peripheral blood according to standard procedures.

Microarray analysis. Genomic screening for copy number variations (CNVs) for patient 1 was performed using Genome-Wide Human SNP Array 6.0 (Affymetrix, High Wycombe, UK). Arrays were analysed using the Scanner 3000 7G and Command Console Software (AGCC) (Affymetrix). Results were processed and visualized by the Genotyping Console software (GTC) 4.0 (Affymetrix). CNV detection was performed using an array-CGH Agilent 105k microarray and an array-CGH Agilent 44k for patients 2 and 3-5, respectively (Agilent, Santa Clara, CA, USA). Male or female genomic DNA (Promega, Charbonnières, France) was used as a reference in sex-match hybridizations, which were analysed on an Agilent scanner with the CGH-analytics software v3.4 (Agilent) by applying Z-score segmentation algorithm with a window size of 10 points to identify chromosome aberrations. In all patients, experiments followed the standard and manufacturer's recommendations. DNA sequence information refers to the public UCSC database NCBI37 (Hg19) (http:// genome.ucsc.edu/cgi-bin/hgGateway) for all patients.

Molecular cytogenetics. Chromosome analysis was performed on R and G banded (550-band level) peripheral blood lymphocytes. CNVs were confirmed by fluorescence in situ hybridization (FISH) analysis using BACs and PACs or commercial $2 \mathrm{p}$ and $2 \mathrm{q}$ subtelomeric probes (Cytocell, Cambridge, UK). The BAC and PAC probes used were DJ-892G20 provided by SJ Knight (Oxford, UK), RP11-1N7 (M Rocchi, Uniba-Bari), and RP11-352J11, RP11-327H5 (N Carter, Sanger Institute, UK) from the telomere to $2 \mathrm{p} 25.2$, respectively.

Parental origin analysis. Genotyping of the DNA from probands 1, 3, 4, and 5 and their parents was performed by PCR amplification using a set of sequencetagged site (STS) microsatellite primers at the 2p25.3 locus: D2S2584, D2S2268, D2S2980, D2S323, and TPOX. The results were analysed with an ABI 3130 Genetic Analyzer (Applied Biosystems, Foster City, CA, USA). Patient 2 inherited the 2 pter deletion as the unbalanced product of a balanced paternal translocation.

\section{RESULTS}

\section{CNV analysis}

Molecular cytogenetic results and microsatellite analyses are summarized in Figures 2 and 3. FISH using a 2ptel subtelomeric probe was 

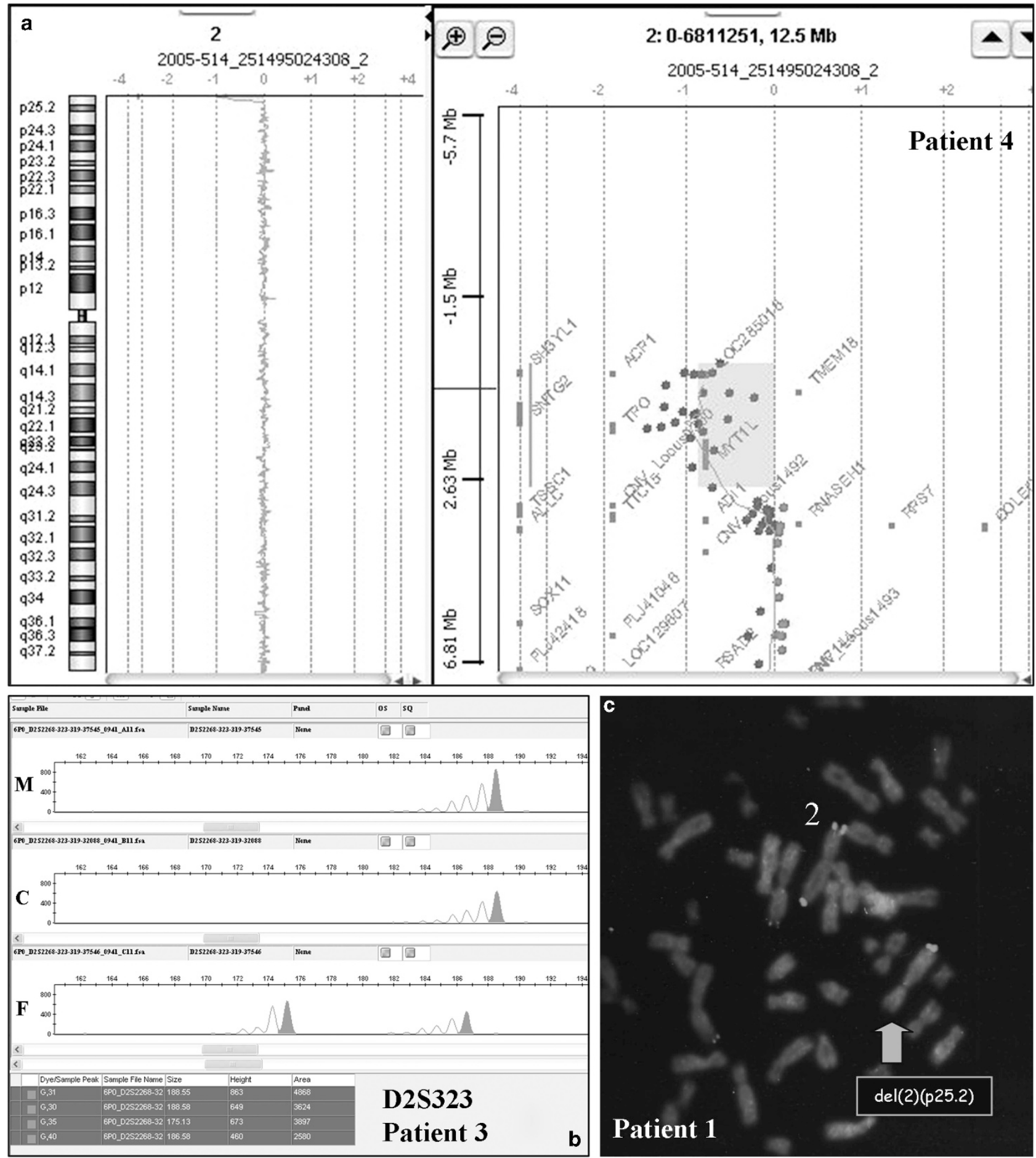

Figure 2 (a) Array-CGH screen shot for patient 4 showing the distal $2 p$ deletion encompassing the subtelomeric region to the MYT1L gene. (b) Genotyping analysis for patient 3 with D2S323 STS showing the absence of the paternal allele for the Child on the second line (C) compared with Mother (M) and Father (F). (c) FISH metaphase for patient 1 with commercial subtelomeric $2 \mathrm{p}$ (green-FITC) and $2 \mathrm{q}$ (red-CY3) probes showing no green signal on the deleted $2 \mathrm{p}$ region.

performed in cases 1-4 showing the deletion (Figure 2). Table 1 summarizes the CNVs observed in the five patients.

Patient 1. Affymetrix SNP arrays showed a $2 \mathrm{p} 25.3$ deletion of a minimum of $1.975 \mathrm{Mb}$ from nucleotide nt 12772 (first deleted probe: CN_865960) to nucleotide nt 1987338 (last deleted probe: SNP_A-8636769): arr 2p25.3 (12772-1987338)x1. The deleted region contains 10 transcribed genes, namely FAM110C, SHY3YL1, ACP1, FAM150B, TMEM18, C2ORF90, SNTG2, TPO, and PXDN genes, and a partial deletion of the MYT1L gene (from telomere to centromere, respectively).

Patient 2. Array-CGH (Agilent 105K) showed a terminal 2p25.3 deletion (chr2: 39193-2397637) with a minimal size of $2.358 \mathrm{Mb}$ : 


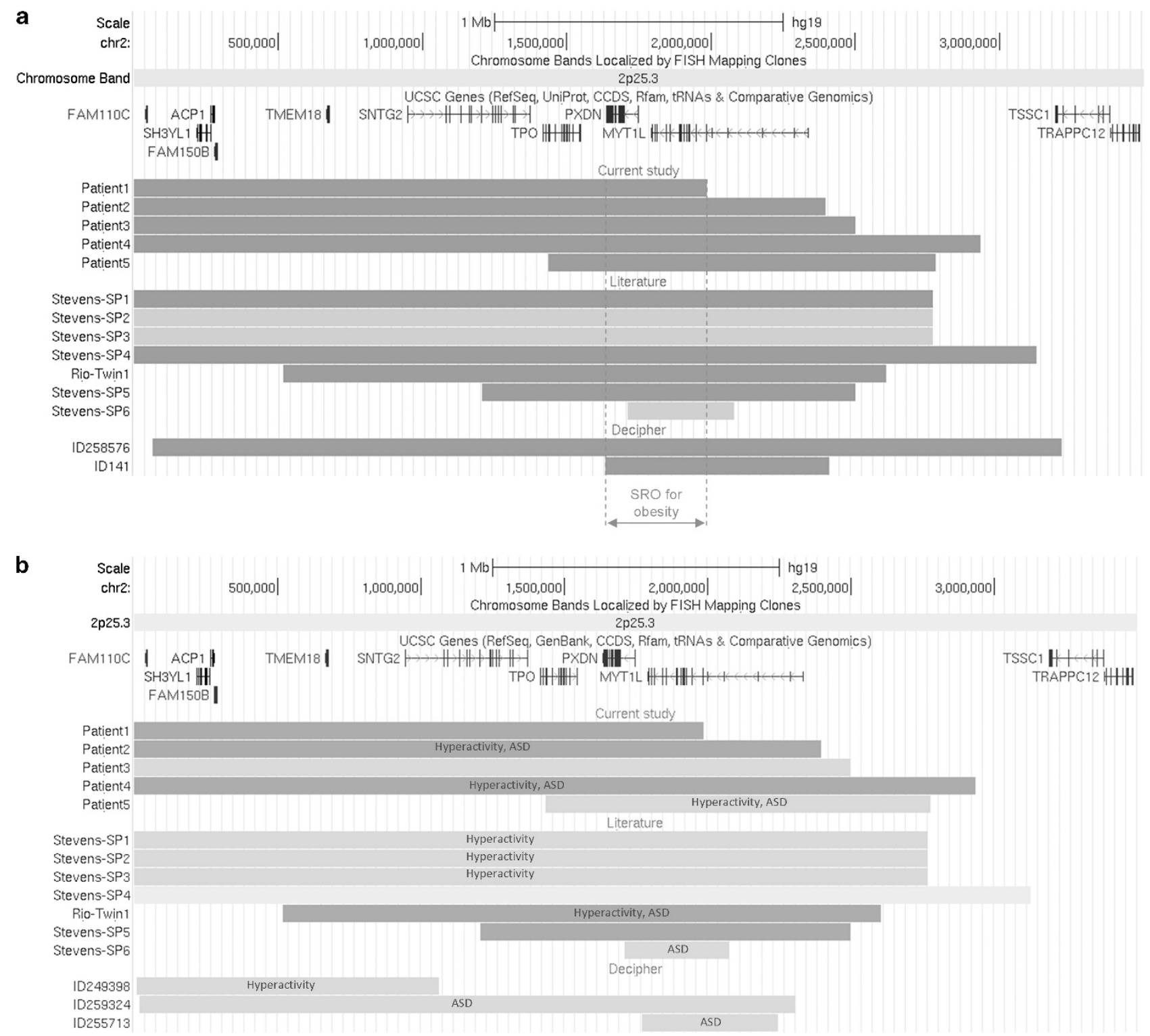

Figure 3 Screen shot UCSC Hg19 Built 37 showing a summary of the deleted segments of patients $1-5$ and the reported patients from Decipher, Stevens et $a l,^{8}$ and Rio et $a l .^{9}$ (a) Correlation map for obesity. In dark grey the obese patients and in middle grey the overweight patients. SRO: smallest region of overlap. (b) Correlation map for behavioural troubles. In dark grey, patients with aggressiveness or outbursts. In middle grey, patients with behavioural troubles missing aggressiveness. In light grey, no reported behavioural troubles. ASD: at least one feature in autism spectrum.

arr 2p25.3 (39 193-2 397637 )x1 (first deleted probe: A_14_P128660 and last deleted probe: A_16_P00301083). The deleted 2p region contains the same 10 genes as described in patient 1 . As this deletion is the result of an unbalanced paternal $\mathrm{t}(2 ; 15)(\mathrm{p} 25.3 ; \mathrm{q} 26.2)$ translocation, array-CGH analysis also showed a 15q26.2-q26.3 duplication of a minimum of $7.373 \mathrm{Mb}$ (chr15: 95021 892-102394710) (first duplicated probe: A_14_P130299 and last duplicated probe: A_16_P20373067).

Patient 3. Array-CGH (Agilent 44K) showed a 2p25.3 deletion of a minimum of $2.461 \mathrm{Mb}$ : arr 2p25.3 (38 993-2 499 660)x1. The deleted region contains the same 10 genes as described in patient 1 .

Patient 4. Array-CGH (Agilent 105K) showed a terminal 2p25.3 deletion of a minimum of $2.895 \mathrm{Mb}$ : arr 2p25.3 (39193-2934684)x1 (first deleted probe: A_14_P133506 and last deleted probe: A_14_P130039).
This result was confirmed by FISH with specific PAC and BAC probes. Parental FISH analysis showed that the deletion arose de novo. FISH: 46,XX.ish $\operatorname{del}(2)$ (pter->p25.3)(DJ-892G20 -, RP11-1N7 -, RP11-352J11 -, RP11-327H5+ )dn. The deleted region contains the same 10 genes as described in patient 1 .

Array-CGH analysis also showed a de novo 13q13.1-q13.1 deletion of a minimal size of $0.93 \mathrm{Mb}(\operatorname{chr} 13$ : 32 127 745-33 055 610).

Patient 5. Array-CGH (Agilent $44 \mathrm{~K}$ ) showed an interstitial 2p25.3 deletion of at least 1.341 Mb: arr 2p25.3 (1437307-2 778661)x1. The deleted region contains the TPO, PXDN, and MYT1L genes.

\section{Parental origin of the deletion}

The deletion was confirmed in patients $1,3,4$, and 5 by the STR study. All informative STS markers lost the paternal 2p25 allele (Figure 2). For patient 2, the 2p25 deleted derivative chromosome was 
inherited as the unbalanced product of a paternal translocation. Consequently, in all patients the paternal 2 p25.3 allele is deleted.

\section{DISCUSSION}

We report on five unrelated patients with a small deletion of the distal part of the short arm of chromosome 2 (2p25.3) of paternal origin and all sharing a common phenotype including early-onset severe obesity and ID associated with behavioural troubles. Among these patients, three have a pure de novo 2 pter deletion, one a cryptic interstitial $2 \mathrm{p}$ deletion, and the last a paternal derivative $\operatorname{der}(2) \mathrm{t}(2 ; 15)(\mathrm{p} 25.3 ; \mathrm{q} 26)$ with deletion in the 2pter region. Interestingly, most of the proximal breakpoints, even if different, are located between MYT1L and TSSC1 as also reported for the largest deletions in Stevens patients (SP1-4). ${ }^{7}$

To the best of our knowledge, partial monosomy 2p25 has been reported in $<20$ patients in the literature ${ }^{2-8}$ (Table 2). The PraderWilli-like phenotype, as reported by Becker $e t a l^{6}$ has been described in the literature in eight other patients with 2 pter deletion. ${ }^{4,7,8}$ Pure 2 pter deletion was reported by Stevens $e a^{7}$ in six adult patients with ID, obesity or overweightness, and/or square-shaped stature. A similar deletion encompassing the FAM110C, SHY3YL1, ACP1, FAM150B, TMEM18, C2ORF90, SNTG2, TPO, PXDN, and MYT1L genes was reported in four out of the six patients, whereas the two remaining patients presented with a smaller deletion encompassing the $T P O$, $P X D N$, and MYTIL genes for one and the PXDN and MYTIL genes for the other. ${ }^{7}$ Rio et al ${ }^{8}$ reported a monozygotic twin displaying ID, obesity, and hyperactivity with a deletion of the TMEM18, SNTG2, $T P O, P X D N$, and MYTIL genes. For the last patients with the PraderWilli-like phenotype (Zou et $a l^{4}$ ), the 2pter deletion was associated with a duplication of another chromosome due to an unbalanced translocation. Parental origin of the 2pter deletion was not reported in Becker's, Stevens', Rio's, and Zou's papers. For the last three patients with 2 pter deletion reported in the literature, clinical information was unavailable. ${ }^{3,5}$ Clinical features of the patients reported in the literature are listed in Table 2.

We found two additional reports in the Decipher database with available clinical features and known obesity and three others with behavioural troubles (http://decipher.sanger.ac.uk/). Figure 3 summarizes the known size of the $2 p$ deletions and the genes encompassed by the deletions observed in this report and those reported in the literature with known breakpoints and phenotype (Stevens et $a l^{7}$ and Rio et $a l^{8}$ ) and from the Decipher database, for obesity or overweight (Figure 3a) and behavioural troubles (Figure 3b).

The most common symptoms among the reported patients are hypotonia (3/5), language delay (5/5), autistic spectrum disorders $(3 / 4)$, behavioural problems $(8 / 8)$, obesity or overweight $(9 / 10)$, and square truncal build (4/6). All the patients reported in the current series share early-onset obesity (5/5), hyperphagia (5/5), and ID associated with behavioural problems (5/5), and some display square truncal build (2/5), small nose (4/5), and small mouth (3/5). In an attempt to determine which genes (or combination of genes) are responsible for the clinical features: overweight/obesity and ID/behavioural troubles, also observed in the patients described in the literature (Table 2), we looked for the known functions of the deleted genes, namely the SH3 domain containing Ysc84-like 1 (SH3YL1), the acid phosphatase 1 soluble (ACP1), the transmembrane protein 18 (TMEM18), the syntrophin gamma 2 (SNTG2), the thyroid peroxidase (TPO), the Drosophila peroxidasin homologue $(P X D N)$ genes, and the myelin transcription factor 1-like (MYT1L) 


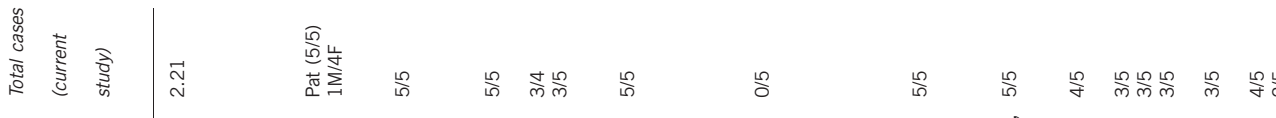

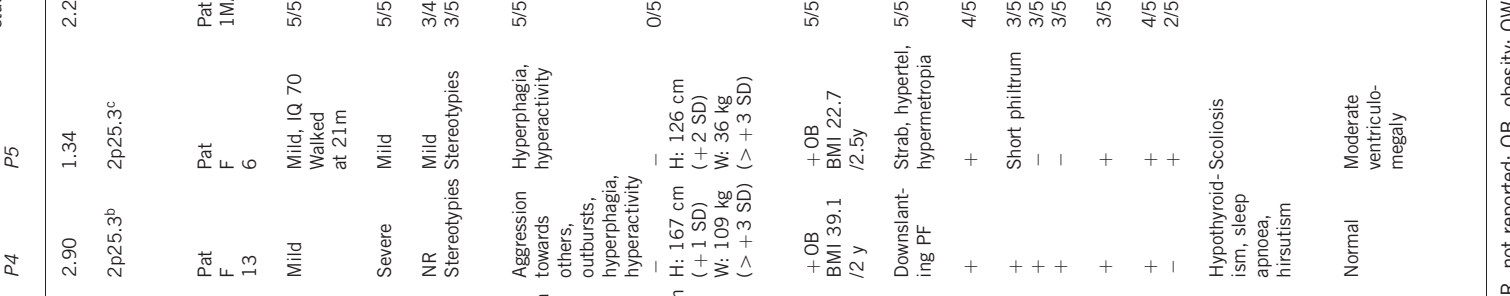

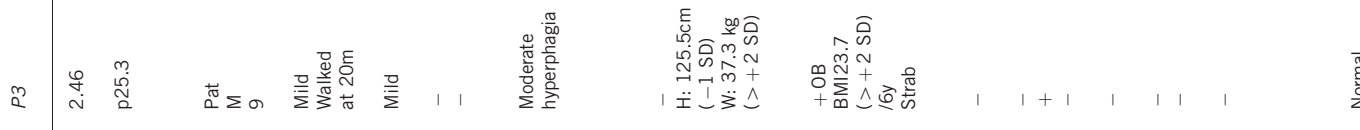

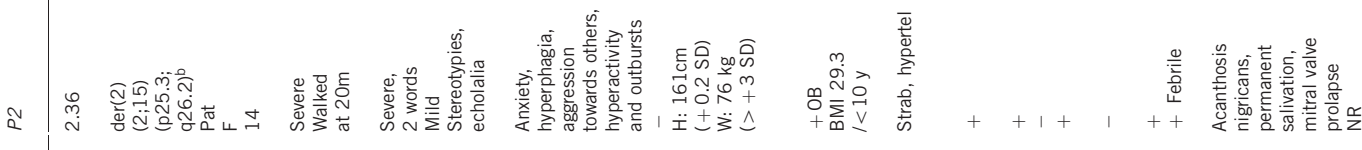

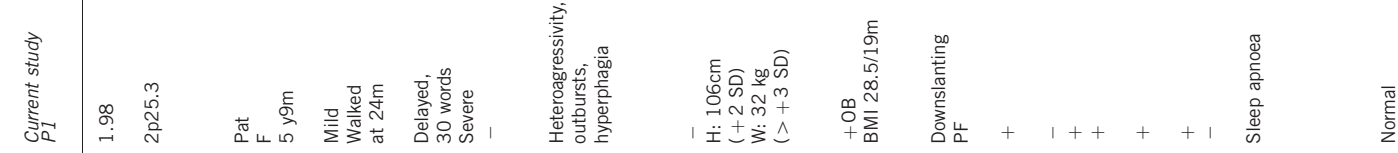

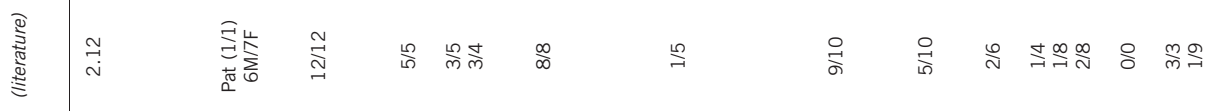

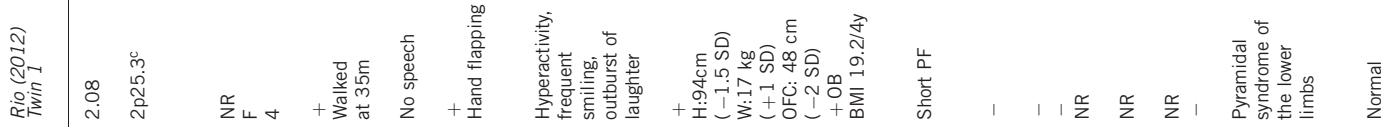

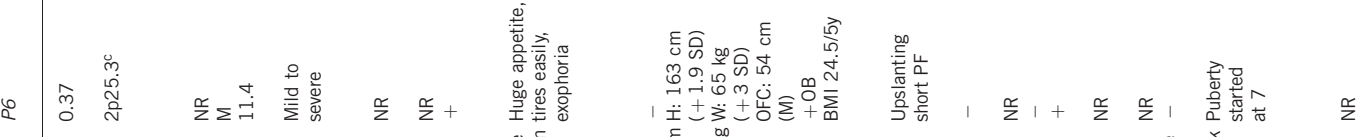

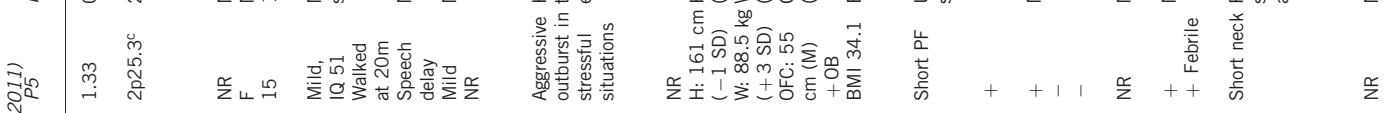

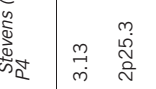

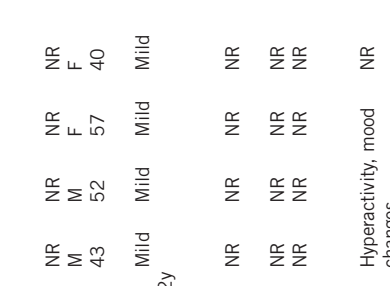

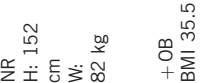

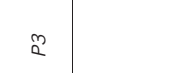

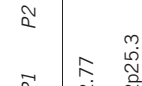

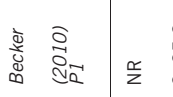

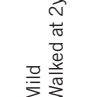

३)

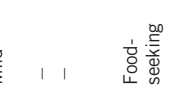

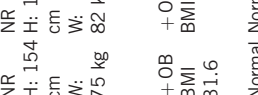

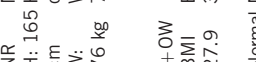

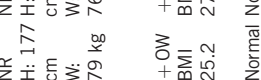

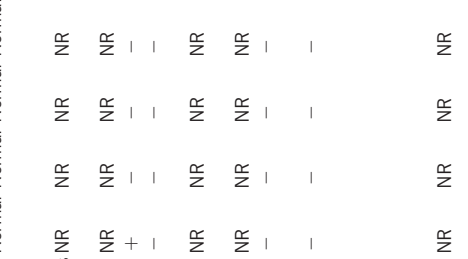

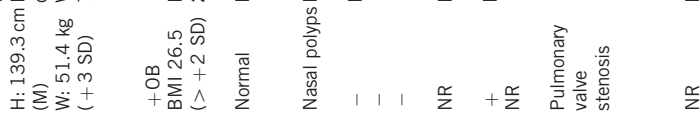

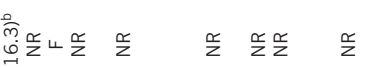

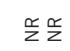

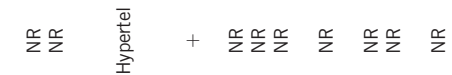

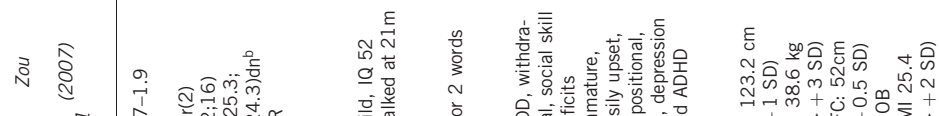

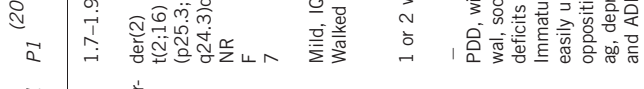

选

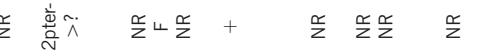

$\frac{\infty}{2} \frac{\infty}{2}$

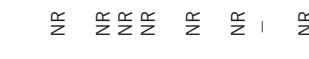

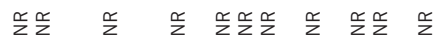

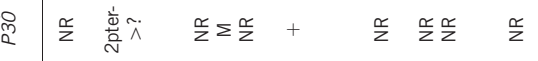

$\frac{\infty}{2} \frac{\infty}{2}$

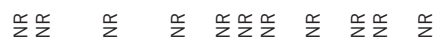

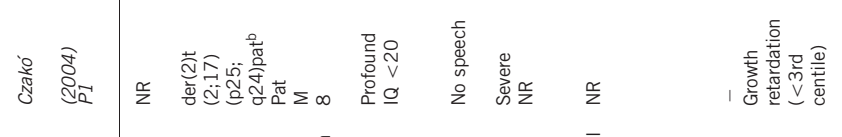

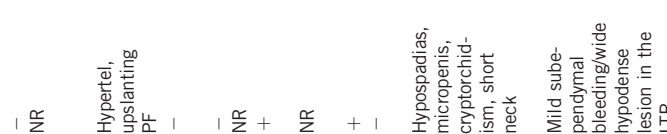


gene previously discussed as the major candidate gene by Stevens et $a l^{7}$ (Figure 3).

\section{Overweight/obesity}

The candidate genes ACP1 and TMEM18 are deleted in four out of the five obese patients of this series (patients 1-4), and in five overweight or obese patients in the literature (SP1-4 and Decipher ID 258576) (Figure 3a). The ACP1 gene is expressed in adipocytes. Polymorphisms in this gene have been associated with severe obesity and with total cholesterol and triglyceride levels. ${ }^{9,10}$ There is an overall positive association between obesity and low activity of acid phosphatase $1,{ }^{10}$ suggesting that the heterozygous loss of $A C P 1$ gene could contribute to the obesity observed in our patients.

The TMEM18 gene is expressed in all brain sites, including the hypothalamus. Genome-wide association studies by the GIANT consortium have shown a direct and significant association between an SNP near the TMEM18 gene and obesity (BMI and weight). ${ }^{11}$ Almen et $\mathrm{al}^{12}$ reported the involvement of TMEM18 in adult and childhood obesity and type 2 diabetes. Nevertheless, the role of haploinsufficiency for TMEM18 is still debated. Possible positional effects are hypothesized by Stevens et al. ${ }^{\text {? }}$

Other genes may be involved as some obese or overweight patients with interstitial deletion 2p25.3 lack neither ACP1 (Twin 1 from Rio et al) nor TMEM18 (P5, SP5, and SP6, Decipher ID 141) (Figure 3a). Furthermore, PXDN and MYT1L genes are located in the smallest region of overlap when looking at the common deleted genes in obese patients with distal or interstitial deletion (Figure 3a). The function of the PXDN gene is not clearly defined in humans. It encodes for a heme-containing enzyme, and is involved in extracellular matrix formation. ${ }^{13}$ PXDN expression was not detected in brain or peripheral blood leukocytes. ${ }^{14}$ MYT1L (see next paragraph) has not yet been described as candidate gene in obesity but six patients (P1-4 and P5, SP6 with interstitial deletion) are deleted for MYT1L and show hyperphagia (Figure 3a).

\section{ID/behavioural troubles}

The candidate genes are SNTG2 and MYT1L. The SNTG2 gene is expressed in brain. This gene is suspected to have a role in autism because its' product interacts with neuroligins NLGN3, NLGN4X, and NLGN4Y, which are autism-related neuroligin isoforms ${ }^{15}$ and because the SNTG2 gene is encompassed in CNVs associated with autism spectrum disorders. ${ }^{16}$ Heterozygous loss of this gene may be responsible for the behavioural troubles observed in our patients. The MYT1L gene encodes for a C2HC-type zinc finger transcription factor highly expressed in developing neuronal cells. ${ }^{17}$ When duplicated this gene has been associated with schizophrenia ${ }^{18,19}$ and major depressive disorder. ${ }^{20}$ Heterozygous loss of function of the MYT1L gene deleted in all our patients could be responsible for the behavioural difficulties and psychomotor retardation described in patients with 2 pter deletion as proposed by Stevens et al. ${ }^{7}$

All patients (P1-5) have mild to severe ID. Behavioural troubles are frequent, namely hyperactivity and mood changes, autistic spectrum disorders, anxiety, and outbursts (Figure 3b). Patients P2, P4-5, SP1-3, Rio's patient and ID 255713 display hyperactivity, and P5 is only deleted for MYT1L. Autistic spectrum disorders are observed in patients with small interstitial deletions encompassing MYT1L such as P5, SP6, and ID 255713. Interestingly, SNTG2 is deleted in P1-2, P4, SP5, and SP6 who display notable aggressivity and outbursts, and is not deleted in P5 and SP6 who do not suffer from aggressiveness. Furthermore, patient ID 249398 from Decipher displays psychosis and hyperactivity with a deletion encompassing partially SNTG2 but not MYT1L, suggesting a potential role of SNTG2 in behavioural troubles. Finally, these troubles might be induced by MYT1L haploinsufficiency and enhanced by the deletion of SNTG2 (Figure 3b).

The TPO gene encodes for thyroid peroxidase, which has an essential role in the process of thyroid hormone synthesis. Impaired TPO function results in iodide organification defect (OMIM 274500) leading to congenital hypothyroidism characterized by a low level of thyroglobulin, low T3 and T4 levels, and elevated blood TSH concentration. ${ }^{21}$ Usually, this condition is inherited in an autosomal recessive manner. ${ }^{22}$ Interestingly, $\mathrm{TSH}$ level was abnormal in $2 / 5$ patients (P1-5) with loss of the paternal TPO gene and paternal monoallelic expression of the TPO gene has been demonstrated in thyroid tissue. ${ }^{23}$

Regarding the parental origin of the deletion, all the patients described here presented with a so-called Prader-Willi-like phenotype (obesity and behaviour troubles) and a paternal deletion. This observation led us to hypothesize a parental effect of the deletion. Furthermore, Czakó et al reported a patient with a paternal 2pter deletion but without the Prader-Willi-like phenotype. In this case, the associated 17q25-qter duplication could mask the phenotype, as ascertained by a rapid review of the literature revealing that patients with 17q25-qter duplication present with severe ID, growth retardation, and facial features resembling those described by Czakó et al. ${ }^{2,24}$ The role of the parental origin of the deletion needs to be further evaluated.

\section{CONCLUSION}

We report five patients with a rare deletion of a small subtelomeric region of the short arm of chromosome $2($ size $<2.90-2.97 \mathrm{Mb})$ in a region containing nine genes. They show hyperphagia with very earlyonset obesity ( $<5$ years of age), ID, and severe behavioural troubles, with aggressive outbursts and hyperactivity. This study confirms that $2 \mathrm{p}$ subtelomeric deletion should be included in the main chromosomal aetiology for syndromic obesity.

\section{CONFLICT OF INTEREST}

The authors declare no conflict of interest.

\section{ACKNOWLEDGEMENTS}

This work was supported by ACLF telomere network (promoter Bordeaux Hospital), the national array-CGH network funded by the French Ministry of Health, and the AOL 2001 project (Reims Hospital), France. This work was supported by grants AOL 2001-2003 CHU Reims and PHRC 2003-2005 CHU-REIMS, and Promoter CHU-Bordeaux France, ACLF réseau télomère. This work was also spported by the grant PHRC N ${ }^{\circ} 2007-A O O 642-51$ CHU Montpellier.

1 Schinzel A: Catalogue of Unbalanced Chromosome Aberrations in Man, 2nd rev. and expanded (edn). Walter de Gruyter: Berlin; New York, 2001

2 Czako M, Riegel M, Morava E, Bajnoczky K, Kosztolanyi G: Opitz 'C' trigonocephaly like syndrome in a patient with terminal deletion of $2 p$ and partial duplication of $17 q$. Am J Med Genet A 2004; 131: 310-312.

3 Ravnan JB, Tepperberg JH, Papenhausen P et al: Subtelomere FISH analysis of 11 688 cases: an evaluation of the frequency and pattern of subtelomere rearrangements in individuals with developmental disabilities. J Med Genet 2006; 43: 478-489.

4 Zou YS, Van Dyke DL, Ellison JW: Microarray comparative genomic hybridization and FISH studies of an unbalanced cryptic telomeric $2 p$ deletion/16q duplication in a patient with mental retardation and behavioral problems. Am J Med Genet A 2007; 143: 746-751.

5 Wu Y, Ji T, Wang J et al: Submicroscopic subtelomeric aberrations in Chinese patients with unexplained developmental delay/mental retardation. BMC Med Genet 2010; 11: 72

6 Becker K, Jaggard C, Horrocks S: A novel presentation of a rare chromosome 2p25.2 deletion. Clin Dysmorphol 2010; 19: 101-102. 
7 Stevens SJ, van Ravenswaaij-Arts CM, Janssen JW et al: MYT1L is a candidate gene for intellectual disability in patients with 2p25.3 (2pter) deletions. Am J Med Genet A 2011; 155A: 2739-2745.

8 Rio M, Royer G, Gobin S et al: Monozygotic twins discordant for submicroscopic chromosomal anomalies in 2p25.3 region detected by array CGH. Clin Genet 2012; 84: 31-36.

9 Paggi A, Borgiani P, Gloria-Bottini F et al: Further studies on acid phosphatase in obese subjects. Dis Markers 1991; 9: 1-7.

10 De Lorenzo A, Di Renzo L, Puja A, Saccucci P, Gloria-Bottini F, Bottini E: A study of acid phosphatase locus 1 in women with high fat content and normal body mass index. Metabolism 2009; 58: 351-354.

11 Thorleifsson G, Walters GB, Gudbjartsson DF et al: Genome-wide association yields new sequence variants at seven loci that associate with measures of obesity. Nat Genet 2009; 41: 18-24.

12 Almen MS, Jacobsson JA, Shaik JH et al: The obesity gene, TMEM18, is of ancient origin, found in majority of neuronal cells in all major brain regions and associated with obesity in severely obese children. BMC Med Genet 2010; 11: 58

13 Peterfi Z, Donko A, Orient A et al: Peroxidasin is secreted and incorporated into the extracellular matrix of myofibroblasts and fibrotic kidney. Am J Pathol 2009; 175: 725-735.

14 Nagase T, Seki N, Ishikawa $\mathrm{K}$ et al: Prediction of the coding sequences of unidentified human genes. VI. The coding sequences of 80 new genes (KIAA0201-KIAA0280) deduced by analysis of cDNA clones from cell line KG-1 and brain. DNA Res 1996; 3 : 321-329, 341-354.

15 Yamakawa H, Oyama S, Mitsuhashi $\mathrm{H}$ et al: Neuroligins 3 and $4 \mathrm{X}$ interact with syntrophin-gamma2, and the interactions are affected by autism-related mutations. Biochem Biophys Res Commun 2007; 355: 41-46.
16 Rosenfeld JA, Ballif BC, Torchia BS et al: Copy number variations associated with autism spectrum disorders contribute to a spectrum of neurodevelopmental disorders. Genet Med 2010; 12: 694-702.

17 Kim JG, Armstrong RC, v Agoston D et al: Myelin transcription factor 1 (Myt1) of the oligodendrocyte lineage, along with a closely related $\mathrm{CCHC}$ zinc finger, is expressed in developing neurons in the mammalian central nervous system. J Neurosci Res 1997; 50: $272-290$

18 Lee Y, Mattai A, Long R, Rapoport JL, Gogtay N, Addington AM: Microduplications disrupting the MYT1L gene (2p25.3) are associated with schizophrenia. Psychiatr Genet 2012; 22: 206-209.

19 Vrijenhoek T, Buizer-Voskamp JE, van der Stelt I et al: Recurrent CNVs disrupt three candidate genes in schizophrenia patients. Am J Hum Genet 2008; 83: 504-510.

20 Wang T, Zeng Z, Li T et al: Common SNPs in myelin transcription factor 1-like (MYT1L): association with major depressive disorder in the Chinese Han population. PLoS One 2010; 5: e13662.

21 Bakker B, Bikker H, Vulsma T, de Randamie JS, Wiedijk BM, De Vijlder JJ: Two decades of screening for congenital hypothyroidism in The Netherlands: TPO gene mutations in total iodide organification defects (an update). J Clin Endocrinol Metab 2000; 85: 3708-3712.

22 Ris-Stalpers $\mathrm{C}$, Bikker $\mathrm{H}$ : Genetics and phenomics of hypothyroidism and goiter due to TPO mutations. Mol Cell Endocrinol 2010; 322: 38-43.

23 Fugazzola L, Persani L, Mannavola D et al: Recombinant human TSH testing is a valuable tool for differential diagnosis of congenital hypothyroidism during L-thyroxine replacement. Clin Endocrinol (Oxf) 2003; 59: 230-236.

24 Lukusa T, Fryns JP: Pure de novo 17q25.3 micro duplication characterized by micro array CGH in a dysmorphic infant with growth retardation, developmental delay and distal arthrogryposis. Genet Couns 2010; 21: 25-34. 